Gdańsk 2020, Nr. 43

https://doi.org/10.26881/sgg.2020.43.10

Paweł Bąk

Universität Rzeszów / Uniwersytet Rzeszowski

ORCID: 0000-0002-7951-6477

\title{
Asymmetrie und Symmetrie in Texten bilingualer Verständigungsdiskurse
}

Der Beitrag knüpft an Untersuchungen der bilingualen Verständigungsdiskurse an und präsentiert bisher nicht besprochene Aspekte dieser Diskurse. Am Beispiel der bilingualen Pressetexte aus der Zeitschrift „Region“" werden Probleme der Symmetrie der Texte besprochen. Bei der Gestaltung der Texte dieses Diskurses wird die strategische Profilierung festgestellt, die die Präsenz der Deutschen und der Polen als Protagonisten des europäischen Geschehens betrifft. Sie treten in Kontexten auf, die für beide Seiten schwierig sind, und werden im besprochenen Diskurs auf eine entsprechend euphemistische Weise in der deutschen und polnischen Sprache dargestellt.

Schlüsselwörter: Hinwendungsstrategie, deutsch-polnischer bilingualer Diskurs, Framing, Profilierung, Translation

Asymmetry and symmetry in texts of bilingual discourses of understanding. - The article continues investigations of the bilingual discourses of understanding and presents aspects not discussed so far. Taking the bilingual press texts from the magazine "Region" as an example, problems of symmetry of the texts are discussed. The strategic profiling that affects the presence of Germans and Poles as protagonists of European events is identified in the design of the texts of this discourse. The Poles and Germans appear in difficult contexts for both societies. In the discourse they are presented in a special way, euphemistically in German or Polish respectively.

Keywords: Strategy of adaptation and positioning, bilingual German-Polish discourse, framing, profiling, translation

\section{Vorbemerkungen}

Seit Jahrzehnten werden im Rahmen der linguistischen Diskursforschung immer neuere Aspekte des sprachlichen, diskursiven Handelns der Menschen unter diversen kommunikativ-medialen Modalitäten erörtert. Im vorliegenden Aufsatz wird auf einige Beobachtungen zu einer spezifischen Profilierung von bilingualen Texten der Verständigungsdiskurse, d. h. der so genannten Hinwendungsstrategie, sowie auf eine für diese Diskurse relevante Kategorie der Erkenntnisperspektive eingegangen. Im Zentrum der Betrachtung stehen bis dato nicht bzw. kaum diskutierte Schnittstellenprobleme zwischen 
Translationswissenschaft und Diskursanalyse einerseits sowie Pragmalinguistik und Semantik andererseits. Sie werden an bisher nicht besprochenen Belegen aus Ausgaben der Zeitschrift „Region“ (Online- und Printausgaben), darunter am Beispielen neueren Datums (nach 2014) beleuchtet.

Der Beitrag knüpft an die bereits eingeleitete Diskussion (Bąk 2010a,b; 2016a,b, 2018a) an, in deren Rahmen die gemeinsame Teilnahme von Deutschen und Polen sowie von anderen Mitwirkenden am politischen Geschehen in Europa untersucht wird, wobei diese Aspekte in einem spezifischen kommunikativen Handlungsrahmen ,bilingualer Verständigungsdiskurse angesprochen werden. Als primäres Charakteristikum dieser Diskurse gilt, dass Autoren der bilingualen Textfassungen sich gegenseitig verstehen und verstanden werden wollen. Unter dem Verständigungsdiskurs wird hier derjenige Diskurs ${ }^{1}$ verstanden, der einen Beitrag zur Aussöhnung zwischen den Deutschen und Polen leisten will. Dabei wird er unter besonderen Umständen und charakteristischen Modalitäten realisiert. ${ }^{2}$

In verschiedenen Diskursen kann man sprachliche Operationen feststellen, durch die dieselben Sachverhalte der außersprachlichen Wirklichkeit in der jeweiligen Darstellung verschiedene Wertung erfahren. Es sind Profilierungen, die durch Rückgriffe auf diverse Wissensbestände des Empfängers, Spiele mit dem Kontext (Dekontextualisierung oder Kontextmodifizierung), metaphorisch-dysphemistische Umdeutungen (Bąk 2019: 30-31) realisiert werden. ${ }^{3}$ Im Folgenden werden sie als strategisches Framing' (Völker 2017: 113-159) aufgefasst, mit dem bewusste, durch Kontextualisierung unterstützte Rückgriffe auf unterschiedliche Wissensressourcen der Sprachbenutzer (z. B. Repräsentanten verschiedener Gesellschaften) ausgeführt werden, die auf bestimmte Bewertungen abzielen.

1 Die bilingualen Verständigungsdiskurse stehen in Opposition zum einsprachigen polnischen Diskurs über Deutschland und dem deutschen Diskurs über Polen. Dem Vorhaben liegen folgende Ansätze zugrunde: die Auffassung des Diskurses nach Foucault (1981), der diskurssemantische Ansatz von Busse (u. a. 2012), Aspekte der Pragma- und Soziolinguistik (u. a. Grice 1975, Goffman 1967, Brown/ Levinson 1987). Unter Diskurs wird in Anlehnung an Foucault (1981: 156) und die an ihn anknüpfende Tradition der Diskursforschung als Bezeichnung für Bündel von Texten und Aussagen verstanden, die dem gleichen Formationssystems angehören (vgl. ebenda und Spitzmüller/ Warnke 2011: 70, 116).

2 Die Spezifik der bilingualen Verständigungsdiskurse besteht u. a. darin, dass der Übersetzer gleichzeitig Koautor der zweisprachigen Ausgabe eines Textes ist, dessen beide Fassungen in ihrer Gesamtheit eine zweisprachige Stimme im Diskurs darstellen wollen. Zur Besprechung der Texte wird angenommen, dass der Übersetzer Autor der zielsprachlichen Fassung ist, der aber gegebenenfalls auch bei der Entstehung des Originals beteiligt sein kann (Bąk 2010a). Beide Autoren bzw. der Autor und Übersetzer sprechen oder verstehen zumindest die jeweils andere Sprache. Dies ist allerdings bei „Region“ nicht immer gegeben, wo nicht nur bilinguale Autoren publizieren.

3 Im Beitrag werden die Kategorien ,Profilierung und ,Framing im Sinn der erkenntnistheoretischen Ansätze der kognitiven Forschung (v.a. von Fillmore 1977, Fraas 2013, Fraas/Meier 2013, Ziem 2013) verwendet. Die ,Profilierung ' wird als eine Art Konzeptualisierung verstanden, in deren Rahmen - entsprechend der Intention der Diskursgestalter - gewisse Aspekte der konstruierten sprachlichen Wirklichkeit aus- oder eingeblendet bzw. hervorgehoben werden (vgl. Czachur 2011: 162-163, 174, 231, 249-255; Pędzisz 2012: 243-245; Ziem 2013: 155, 159-163; Bąk 2019: 29-31). 
In bilingual ${ }^{4}$ verfassten Texten des untersuchten Verständigungsdiskurses, die die beiden Völker in gegenseitiger Berührung betreffen, wird auf strategisches Framing (Völker 2017: 113-159) zugunsten einer Profilierung verzichtet, die beide diskursiven Parteien im verträglichen Kommunikationsmodus ansprechen und so den deutsch-polnischen Beziehungen zu friedlicherem Nebeneinander verhelfen sollte. ${ }^{5}$

Im Rahmen des gesamten Forschungsvorhabens, dessen Teil der vorliegende Beitrag ist, werden am Beispiel der zweisprachigen Presse („Region“ und „Dialog“) sowie an gemeinsamen Erklärungen der deutschen und polnischen Bischofskonferenzen (seit 1995) bisher kaum erforschte Probleme der bilingualen öffentlichen Verständigungsdiskurse (Bąk 2016a,b) diskutiert. ${ }^{6}$ Die Aspekte betreffen die (A)Symmetrien von Original und Translat. Diese Probleme wurden bereits in anderen Studien gestreift, in denen allgemeine Merkmale der bilingualen Diskurse am Beispiel der Zeitschrift „Region“ (Bąk 2010a) und der Erklärungen (Bąk 2010b) präsentiert wurden. Die hier umrissenen Aspekte beruhen auf einem sprachlichen Material, das aus anderen Anwendungsgebieten stammt und gewissermaßen alltagsnäher ist als die von deutschen und polnischen Bischöfen gemeinsam verfassten Texte. Nichtsdestoweniger bestätigen die hier angeführten Belege die Beobachtungen, die anhand anderer Texte des bilingualen Verständigungsdiskurses bisher gemacht wurden.

\section{Symmetrie und Asymmetrie von Sprachversionen der Texte des bilingualen Verständigungsdiskurses}

\subsection{Formal-strukturelle Asymmetrie der Texte}

Zwischen den beiden Sprachversionen der untersuchten Texte sind diverse, oft auf den ersten Blick banale Abweichungen in der Grammatik bzw. Lexik festzustellen, die durch sprachsystematische Unterschiede bedingt, auf abweichende Konzeptualisierung bzw. auf deren divergierende sprachliche Realisierung in beiden Sprachen zurückzuführen sind. Solche Unterschiede ((1) und (2)) haben zumeist grammatischen und lexikalischen Charakter. ${ }^{7}$

(1) Republika Federalna Niemiec, która wyłączyła z rywalizacji w krajowych kwalifikacjach w roku 1959 drużynę NRD, przegrała wynikiem 0:3 w pierwszym meczu z Polską rozegranym 29 listopada 1959 w Essen. (A 1/2016 poln. online)

4 Der in Bezug auf die untersuchten Diskurse verwendete Ausdruck ,bilingual' ist nicht nur die Bezeichnung der äußeren Realisierungsform der Texte, sondern auch der Einbettung der Sprachen und Diskurse in den beiden Kulturräumen und Denkkulturen.

5 In einem anderen Zusammenhang (Bąk 2020 im Druck) wird an weiteren Belegen exemplifiziert, wie die Diskursgestalter im Rahmen der Hinwendungsstrategie dem polnischen Leser durch Attribuierungen die Realien Deutschlands näherbringen wollen (Bąk 2020 im Druck). Damit kann die negative Wirkung des Framing verhindert werden.

6 Die Texte stellen als Bestandteile des deutsch-polnischen Verständigungsdiskurses den Gegenstand der Untersuchung des von der Alexander-von-Humboldt-Stiftung seit 2017 geförderten Projektes „Die Strategie der Hinwendung in bilingualen Verständigungsdiskursen " dar.

7 In vielen Fällen hat man es mit der von Sprache zu Sprache unterschiedlichen Valenz und Rektion zu tun. 
(1') Die Bundesrepublik, die in einer innerdeutschen Quali 1959 schon die DDR ausgeschaltet hatte, vergeigte das Hinspiel gegen Polen am 29. November 1959 in Essen mit 0:3. (A 1/2016 dt. online)

(2) Tak więc tylko dwóch dziennikarzy towarzyszyło drużynie podczas meczu rewanżowego w Warszawie, który nie pozostawiał właściwie żadnych szans. (A 1/2016 poln. online)

(2') So begleiteten nur noch zwei Journalisten das Team zum eigentlichen aussichtslosen Rückspiel nach Warschau. (A 1/2016 dt. online)

In der Konstellation der untersuchten Texte der bilingualen Diskurse manifestieren sich solche Divergenzen in einer Asymmetrie. ${ }^{8}$ Hinsichtlich der sprachsystematischen Unterschiede zwischen Sprachen fallen bei der Analyse besonders quantitative Abweichungen zwischen den beiden Textfassungen auf. Sie können im translationswissenschaftlichen Rahmen als obligatorische Transpositionen erörtert werden. ${ }^{9}$ Die Herausforderung bei jeder Übersetzung, mag es sich dabei um die Übersetzung aus dem Polnischen ins Deutsche oder umgekehrt, aus dem Deutschen ins Polnische handeln, ist primär mit der Notwendigkeit verbunden, formalsystemische Differenzen zwischen den beiden Sprachen zu überwinden. In der Übersetzung zwischen dem Deutschen und Polnischen manifestieren sich die sprachtypologisch bedingten Divergenzen u. a. im morphosyntaktischen Bereich, d. h. in der stärker ausgeprägten Tendenz zum analytischen Sprachgebrauch im Deutschen. Auch im hier untersuchten Diskurs kann dies beobachtet werden, allerdings fallen hier solche Abweichungen nicht so stark wie bei der Übersetzung von Kurztexten ins Auge (Bąk 2018b). Den polnischen synthetischen Sprachstrukturen entsprechen Formulierungen der deutschsprachigen Version, in denen die lexikalisch-grammatischen Funktionen auf mehrere freie Morpheme verteilt sind.

(3) Książka, którą napisałem, przedstawia nie tylko historię bankowości czy mechanizmy regulujące tworzenie i pożyczanie pieniądza [...]. (B 1/2016 poln. online)

(3) Das Buch, das ich geschrieben habe, schildert nicht nur die Geschichte des Bankwesens oder die Regulierungsmechanismen für die Geldschöpfung und die Kreditvergabe [...]. (B 1/2016 dt. online)

(4) Reszta [dziennikarzy - P.B.] traci później krytycyzm. (C 1/2014 poln. online)

(4) Den anderen [Journalisten - P.B.] kommt anschließend ihre kritische Haltung abhanden. (C $1 / 2014$ dt. online)

(5) Dzięki bezkarności elit sztucznie poprawiane wskaźniki makroekonomiczne coraz bardziej rozmijają się z realiami dnia codziennego i ocenami zwykłych ludzi. (B 1/2016 poln. online $)^{10}$

(5) Durch Eliten, die nie juristische Folgen zu fürchten hatten, wurden die makroökonomischen Indikatoren schön gerechnet und gehen an der Realität und den Erwartungen der Bevölkerung völlig vorbei (sic!). (B 1/2016 dt. online)

8 Asymmetrien sind besonders bei Abweichungen der konzeptuellen Metaphorik, der metaphorischen Phraseologie oder im strukturellen, morphosyntaktischen Bereich sichtbar (Bąk 2010b). Im vorliegenden Beitrag wird aus Platzgründen nicht auf Unterschiede eingegangen, die in der Metaphorik und der metaphorischen Phraseologie vorkommen.

9 Das Verhältnis zwischen der Wörtlichkeit und der Modifizierung der übersetzten Texte drückt sich in der Literatur u. a. in der Unterscheidung zwischen direkten Übersetzungstechniken und Transpositionen aus, d.h. in der Auffassung von modifizierenden Prozeduren als fakultative oder obligatorische Transpositionen. Es sind also Verfahren, die der Übersetzer durchführen muss oder kann. Bąk (2018b: 123-137), vgl. aber auch andere Ansichten in Vinay/Darbelnet (1958: 50) und Schreiber (1993: 214).

10 Bei diesem Beleg kommt die Frage nach dem obligatorischen Charakter der vorgenommenen translatorischen Transposition auf. 
Wie in einem anderen Zusammenhang geschildert wurde (Bąk 2018b), sind oft (nicht nur im bilingualen Pressediskurs) Asymmetrien zwischen Sprachen des Originals und der Übersetzung auf der morphosyntaktischen Ebene zu sehen. Abgesehen davon, dass in der deutschen Textversion manchmal als äquivalentes Vergangenheitstempus das von der Form her kompaktere Präteritum Aktiv verwendet wird, können sprachtypologisch bedingte Unterschiede zwischen Sprachen besonders beim Vergleich von exzerpierten einzelnen Sätzen und Textabschnitten deutlich werden. ${ }^{11}$ Anhand des untersuchten Korpus des Verständigungsdiskurses können jedoch auch Gegenbeispiele angeführt werden, d.h. Belege dafür, dass den deutschen kompakten Wortbildungsformen (Komposita) polnische freie bzw. lose Wortgruppen oder Wortgruppenlexeme entsprechen.

(6) [...] w określonych sądach [są oni] jak np. w Berlinie czy w Hamburgu, z reguły lepiej traktowani. $\mathrm{Z}$ uwagi na to, że prawo do prywatności w tych miastach jest tak restrykcyjnie przestrzegane, artykuł zawierający podejrzenie popełnienia przestępstwa jest prawie niedopuszczalny. (I 1/2009: 6 poln./dt. Druck)

(6) Und sie haben bei bestimmten Gerichten wie in Berlin oder Hamburg in der Regel die besseren Karten. Denn dort wird das Persönlichkeitsrecht so hoch gehalten, dass eine Verdachtsberichterstattung fast unmöglich geworden ist. (I 1/2009: 8 poln./dt. Druck)

(7) Od wczesnej młodości uprawiałem intensywnie sport, przez długi czas żyłem wstrzemięźliwie i dopiero praca w redakcji gazety powojennej, której pracownicy nie oszczędzali się w spożyciu piwa i wódki, wprowadziła mnie w życie alkoholika. (A 1/2016 poln. online)

(7`) Ich hatte seit frühester Jugend intensiv Sport getrieben, lange asketisch gelebt, und erst die Arbeit in den Bier-und-Schnaps-intensiven Zeitungsredaktionen der Nachkriegszeit bereitete mich auf ein Alkoholikerleben vor. (A 1/2016 dt. online)

Insgesamt kann man festhalten, dass sich an mehreren Textstellen der analysierten Artikel die sprachsystematisch bedingten, grammatisch-lexikalischen Erweiterungen bzw. Reduzierungen ausgleichen, so dass die beiden Sprachfassungen trotz vorhandener Divergenzen keine übertriebenen, gravierend quantitativen Unterschiede aufweisen (Ausbau der deutschen Phrase trotz der Komposition). Nichtsdestoweniger liegen in solchen Fällen sprachstrukturelle Asymmetrien von Ausgangs- und Zieltexten vor.

(8) Pierwszy raz w życiu „urwał mi się film”. (A 1/2016 poln. online)

(8) Ich hatte zum ersten Mal in meinem Leben einen „Filmriss”. (A 1/2016 dt. online)

(9) Dlatego teraz tylko w telegraficznym skrócie: rzekome dzienniki Hitlera widziałem u pewnego kolekcjonera w okolicach Stuttgartu. (D 1/2016 poln. online)

(9`) Deshalb hier nur im Telegrammstil: Ich sah ein angebliches Hitler-Tagebuch bei einem Sammler in der Nähe von Stuttgart. (D 1/2016 dt. online)

In der Analyse der Texte des bilingualen Verständigungsdiskurses sind jedoch außer den durch sprachsystematische Unterschiede erzwungenen Veränderungen der Textgestalt wichtigere, weil vom Übersetzer aus eigenem Antrieb vorgenommene Eingriffe in die Textgestalt, zu beachten. Bei solchen Veränderungen (Beleg (5)) sind nicht nur der fakultative oder obligatorische Charakter der Änderung, sondern auch ihre Wirkung und v.a. pragmatische Gründe

11 Belege sind in Bąk (2018b: 130) zu finden. 
für mehr oder weniger wesentliche Modifizierungen ${ }^{12}$ der zielsprachlichen Textstruktur zu hinterfragen. Aus translationswissenschaftlicher Sicht haben sie fakultativen Charakter und in diskursanalytischer Hinsicht sind sie im Rahmen einer bewusst verfolgten Übersetzungsund Textgestaltungsstrategie zu betrachten.

(10) Praca polegająca na szyciu tapicerek do luksusowych mercedesów była lekka jak życie w nowej ojczyźnie. (E 1/2016 dt. online)

$\left(10^{\circ}\right)$ Sie nähte Sitzbezüge für luxuriöse Limousinen - die Arbeit und das Leben in der neuen Heimat waren einfach. (E 1/2016 dt. online)

(11) Dziennikarze, którzy zajmują się tzw. trudnymi tematami, wpadają często w wir zaskakujących wydarzeń, który pochłania ich, a niekiedy niszczy. (D 1/2016 poln. online)

(11') Journalisten, die sich mit schwierigen Themen befassen, geraten häufig in einen Strudel von Ereignissen, die durchaus auch zerstörerisch sein können. (D 1/2016 dt. online)

Im Falle der bilingualen Diskurse hat man es mit einer besonderen Konstellation und einem besonderen Status des Autors sowie des Übersetzers zu tun. Letztere sind gleichzeitig Koautoren der zweisprachigen Ausgabe eines Textes, dessen beide Versionen in ihrer Gesamtheit eine zweisprachige Stimme im Diskurs bilden. Der Übersetzer/Autor der zielsprachlichen Fassung kann auch an der Entstehung des Originals beteiligt sein bzw. er kann die letztere beeinflussen.

Bei der Erklärung des Phänomens der Symmetrie und Asymmetrie in bilingualen Verständigungsdiskursen sind die in Bąk (2016a,b, 2018a, 2019) diskutierte Erkenntnisperspektive (2.2) sowie die bereits 2010 geprägte Kategorie der Hinwendungsstrategie (Bąk 2010a, 2016a,b, 2018a) aufschlussreich (s. 2.3).

\section{Symmetrie der Texte des bilingualen Verständigungsdiskurses}

\subsection{Hinwendung als natürliche Erscheinung in bilingualen Texten}

In den im Rahmen des Projektes untersuchten bilingualen Diskursen ${ }^{13}$ werden Symmetrien zwischen den beiden Sprachversionen der Texte festgestellt. Zum einen ist die Symmetrie eine nichtstrategische, natürliche, sozusagen selbstverständliche Relation und das Ergebnis der jeweiligen Perspektive des Sprechers, eines Polen oder eines Deutschen. Es ist nicht etwa das Resultat einer überdachten diskursiven Taktik, eine auf spezifische Weise profilierte Sprechhandlung zu vollziehen (vgl. Bąk 2016a: 252-253; 2016b: 17). In diesem Zusammenhang sind deiktische Ausdrücke, Kollokationen (im weiteren Sinn verstanden) sowie Nominationsstereotype (Fleischer 1997: 58-62) zu nennen, die die spiegelbildliche Konstellation der zwei Sprachvarianten von Texten der bilingualen Diskurse herstellen:

\footnotetext{
12 Fakultativ erscheinen Eingriffe in den Text, die Symptom eines schöpferischen Engagements des Translators sind. Üblicherweise werden sie von Übersetzungskritikern gelobt (vgl. dazu Bąk 2018b).

13 Dies betrifft nicht nur die bilinguale Presse, sondern auch gemeinsame Erklärungen von Repräsentanten der deutschen und polnischen Kirche (Bąk 2010b, 2016a,b).
} 
(12) Trzydziestokilkuletni Niemcy [...] [o]dpowiadali zawsze w ten sam sposób: My tu co najwyżej przyjedziemy pozwiedzać [...]. (H 2/2009: 16 poln./dt. Druck $)^{14}$

(12') [D] reißigjährige Deutsche [...] antworteten alle dasselbe: Wir fahren höchstens als Touristen oder als Geschäftsleute hin. (H 2/2009: 17 poln./dt. Druck)

In Begleitung des Verbs mit dem Präfix przy- (,an-', ,heran-', ,her-') hat das Adverb tu (,hier', ,her') richtungsweisenden Charakter. Im Satz wird dadurch die Perspektive der Betrachtung der Richtung, nach Polen her' gestaltet. Dieser Perspektive entspricht in der deutschen Fassung ein entgegengesetzter Blickwinkel (,nach Polen hin'). In die verbal auf diese Weise profilierte Konstellation können mit den beiden Sprachversionen sowohl der deutschsprachige als auch der polnische Leser mit einbezogen werden. Beide Rezipientenkreise können sich auf diese Weise besser angesprochen fühlen.

Die Symmetrie von Original und Translat wird hier (und in vielen anderen Belegen (Bąk 2010a,b)) durch den Einsatz von deiktischen Ausdrücken ermöglicht, mit denen auf nichtsprachliche Größen, hier auf räumliche Bezugspunkte, hingewiesen wird.

An einer anderen Textstelle gestaltet der Autor/Übersetzer die Perspektive aus eigenem Antrieb so, dass außer dem Adverb tam (,dort') (13) eine ausgebaute Struktur zu finden ist, für die im deutschsprachigen Wortlaut $\left(13^{\circ}\right)$ keine Entsprechung vorliegt. Seine Entscheidung, den Satz in der deutschen Version auszulassen, kann als eine radikale und zugleich kontroverse Lösung angesehen werden. Anzumerken ist dabei, dass es sich hier zugleich um das Ergebnis einer bewussten und ernst genommenen Strategie der Textgestaltung handelt. Die asymmetrische Profilierung entspringt einer durchdachten diskursiven Strategie. Dabei hat sie im Vergleich zur im untersuchten Diskurs erkennbaren natürlich-symmetrischen Deixis einen stärker pragmatischen Charakter. ${ }^{15}$

Die in (13) und (13') stark asymmetrische Profilierung von Aspekten, die die deutschpolnischen Beziehungen betreffen (Auslassung der gesamten letzten Phrase in der deutschen Fassung), ermöglicht es, die beiden Parteien, d.h. die Deutschen und die Polen, in der jeweiligen Textversion im positiven Licht erscheinen zu lassen. Einer weiteren Diskussion bedarf allerdings im translationskritischen Rahmen die Legitimität dieser Technik wegen des an Manipulation grenzenden Eingriffs des Übersetzers in die deutschsprachige Textversion.

(13) Deklarowanie, że jakaś niemiecka partia będzie zabiegać o interesy niemieckich wypędzonych, to również ukłon wobec prawicowego wyborcy, zorientowanego na powrót silnych Niemiec. Tacy przecież też tam są - co nikogo nie powinno dziwić. (H 2/2009: 16 poln./dt. Druck)

(13’) Die Ankündigung einer deutschen Partei sich für die Interessen der Vertriebenen einzusetzen ist auch eine Geste an rechte Wähler, die an einem wiedererstarkenden Deutschland interessiert sind. (H 2/2009: 17 poln./dt. Druck)

Veränderungen einer der Textfassungen - wie die zuvor herangezogene $\left(13^{\circ}\right)$ - werden im Rahmen der hier besprochenen Strategie der Anpassung bzw. Zuwendung zum

14 Für diesen und weitere Belege sowie für weitere Beispiele für den deiktischen Sprachgebrauch Bąk (2016a: 253).

15 Mehr zu diesem Beleg in Bąk (2010a: 157). 
zielsprachlichen Leser (Hinwendung zum Adressaten) erörtert, die als Hinwendungsstrategie bezeichnet wird (Bąk 2010a, 2016a,b, 2018a).

\subsection{Erkenntnisperspektive}

Bevor auf Belege für die Gestaltung der Symmetrie im bilingualen Verständigungsdiskurs (2.3) im Zusammenhang mit dem diskursspezifischen Paradigma des Sprechenden und des Angesprochenen eingegangen wird, sei kurz am Beispiel der schematischen Adjektivverbindungen deutsch-polnisch im Deutschen und polsko-niemiecki (,polnisch-deutsch') im Polnischen die Kategorie der Erkenntnisperspektive angesprochen. Die beiden Nominationsstereotype polsko-niemiecki und deutsch-polnisch sind in diesen Formen in den beiden Sprachen gebräuchlicher als die kaum geläufigen, unnatürlichen Verbindungen polnisch-deutsch oder niemiecko-polski (,deutsch-polnisch'). ${ }^{16}$

(14) Kongres Polonii Niemieckiej powstał w lutym 1992 roku, aby reprezentować interesy Polonii wobec władz polskich i niemieckich na mocy wspomnianego Traktatu o Przyjaźni i Współpracy Polsko-Niemieckiej z 1991 roku. (I 1/2009: 14 poln./dt. Druck)

(14') Der Kongress der Auslandspolen in Deutschland entstand im Februar 1992, um die Interessen der Polen gegenüber polnischen und deutschen Behörden auf Grundlage des genannten Abkommens über freundschaftliche deutsch-polnische Zusammenarbeit von 1991 zu vertreten. (I 1/2009: 15 poln./dt. Druck)

(15) Wydawało się, że „wojna” o E. Steinbach prowadzona przez prof. Bartoszewskiego, a następnie bardzo mocne potraktowanie jego osoby przez niemieckie media było ostatnim akordem w trudnych relacjach polsko-niemieckich. Okazało się niestety, że nie. (H 2/2009: 12 poln./dt. Druck)

(15’) Es schien, als wäre Prof. Bartoszewskis Krieg um Erika Steinbach und die anschließende recht aggressive Abfuhr seitens deutscher Medien der letzte und kräftigste Akkord der schwierigen deutsch-polnischen Beziehungen. Doch es zeigte sich, dass es leider nicht so war. (H 2/2009: 13 poln./dt. Druck)

Die im Hinblick auf die Reihenfolge der Attributsadjektive vergleichsweise feste Verbindung, die im deutschen und im polnischen Sprachgebrauch in der zueinander umgekehrten Reihenfolge vorkommen, spiegelt die Erfahrung des Sprachgebrauchs sowie die Erfahrung der Betrachtungsperspektive von verbalisierten Ausdrücken wider. Dies bedeutet, dass nicht nur die Ausdrücke, sondern auch die Perspektive den Gegenstand der Erkenntnis und der Erfahrung darstellt. Aus diesem Grund sollte in diesem Zusammenhang nicht von der Betrachtungsperspektive als Kategorie rein pragmatischen Charakters die Rede sein, sondern von der Erkenntnisperspektive. ${ }^{17}$

An dieser Stelle möchte ich zumindest kurz auf die in diesem Beitrag vorausgesetzte Sichtweise der Semantik eingehen. Die Bedeutung verstehe ich (Bąk 2019: 31-32) als eine Entität, die konkrete Erkenntnisakte und Aktualisierungen von Wissensbeständen der Sprachbenutzer

16 Dies kann man anhand des Vorkommens der Wortverbindungen im zweisprachigen Diskurs sowie anhand der Treffer in Suchmaschinen wie Google (www.google.de und www.google.pl) feststellen (Bąk 2016b: 17). Diese Problematik wurde an Belegen aus anderen Quellen in Bąk (2018a) ausführlicher diskutiert.

17 Mehr zu diesem Vorschlag in Bąk (2019: 32-37). 
konstituiert (ebd. und Busse 2012: 11-25, Pawłowski 2017: 52). Bedeutung ist also ein semantisches, durch verschiedene kommunikativ-pragmatische Faktoren, jedoch vor allem durch individuelles Wissen geprägtes Interpretationskonstrukt. Die Konstituierung der Bedeutung erfolgt durch Bezug auf das Wissen des Individuums, seine Wissensbestände, die wiederum durch Erfahrung des Sprachgebrauchs entwickelt werden. Besonders wichtig sind dabei sein durch Erfahrung erworbenes Wissen (Sprach- und Weltwissen), die Rolle im Diskurs (z. B. der Sprechende oder Angesprochene), die Kenntnis des textsorten- bzw. diskursspezifischen Sprachgebrauchs und nicht zuletzt auch die Erfahrung der Erkenntnisperspektive, dank welcher Verbindungen wie deutsch-polnisch und polsko-niemiecki zu Nominationsstereotypen werden können.

Die oben angerissenen Beobachtungen sind dank der für den bilingualen Diskurs spezifischen, von Autoren/Übersetzern konsequent verfolgten Strategie der Gestaltung des Diskurses möglich, in der der adäquate Sprachgebrauch, die Präsenz und der Status von Diskursakteuren und Textadressaten übereinstimmen sollten.

Eine anders gestaltete Perspektive (etwa auf Polnisch ,niemiecko-polski ' und auf Deutsch ,polnisch-deutsch') könnte, ähnlich wie beim Funktionieren des Mechanismus der Konversationsmaximen (Grice 1975), als Verstoß gegen die „Ordnung“ des bilingualen Diskurses gelten und wichtige Informationen über die kommunikative Intention und Einstellungen der Sprecher zu den besprochenen Personen und Themen liefern. ${ }^{18}$ In den untersuchten Texten kann man beobachten, dass andere Protagonisten des Diskurses (Amerikaner, Ukrainer oder - wie nachstehend - Russen) aus der Hinwendungsstrategie ausgeklammert sind. Im untersuchten Diskurs erscheinen sie nicht wie die Sprechenden und Angesprochenen in einem Gespräch, sondern als Besprochene. ${ }^{19}$ Ist in den Texten etwa von Beziehungen zu Russland die Rede, dann gestaltet sich die Konstellation der beiden Sprachfassungen nicht mehr symmetrisch: (deutsch-russisch, niemiecko-rosyjskie).

(16) kontakty niemiecko-rosyjskie, dzięki staraniom wielu pokoleń polityków, osiągnęły „wyjątkową jakość". (I 1/2009: 28 poln./dt. Druck)

(16') Die deutsch-russischen Beziehungen konnten dank vieler Generationen von Politikern eine „besondere Qualität“ erreichen. (I 1/2009: 29 poln./dt. Druck)

(17) „Gazociąg ma służyć interesom niemieckim i rosyjskim. Nie rozumiem, co w tym złego.” (I 1/2009: 28 poln./dt. Druck)

(17 ) Die Gaspipeline soll deutschen und russischen Interessen dienen. Ich kann nicht verstehen, was daran schlecht sein soll - fuhr der Bundeskanzler fort. (I 1/2009: 29 poln./dt. Druck)

(18) W czasie spotkania z dziennikarzami, Schröder zapewniał, że porozumienie o budowie niemieckorosyjskiego gazociągu nie jest wymierzone w inne kraje, „ani w kraje bałtyckie, ani też w Polskę”. (I 1/2009: 28 poln./dt. Druck)

(18') Bei einem Treffen mit Journalisten versicherte Schröder, dass sich das Abkommen zum Bau der deutsch-russischen Pipeline nicht gegen andere Länder richtet, „weder gegen die baltischen Länder noch gegen Polen“. (I 1/2009: 29 poln./dt. Druck)

18 Eine andere Reihenfolge der Glieder der schematischen Adjektivverbindung kann - auch in anderen Texten (Bąk 2018a: 55-57) - durch weitere pragmatisch-inhaltliche Gründe bedingt sein, die hier aus Platzmangel nicht diskutiert werden können.

19 S. zu diesem Paradigma nachstehend 2.4. 
Die Reihenfolge der Sprachbezeichnungen erscheint in ähnlich gebildeten sprachlichen Strukturen in untersuchten Texten des bilingualen, deutsch-polnischen Verständigungsdiskurses als Mechanismus und Instrument der Profilierung. Ihre konsequente Verfolgung ermöglicht die Realisierung der diskursiven Strategie der Hinwendung (kurz: Hinwendungsstrategie). Die Profilierung entspricht der jeweiligen Perspektive oder dient der Herstellung der jeweiligen „deutschen“ oder „polnischen“ Perspektive. Damit ist die Logik der Präsenz von Diskursakteuren verbunden, also die Art und Weise, wie letztere in Texten angesprochen werden.

\subsection{Strategie der Hinwendung zum Leser: der Sprechende und der Angesprochene}

In Bąk (2010a) wurden Belege aus der bilingualen Presse, die vor 2010 erschienen ist, untersucht, wobei die Texte gemeinsame Probleme ${ }^{20}$ der Deutschen und der Polen in deren gegenseitiger Berührung thematisieren. Es wurde gezeigt, dass die Texte inhaltlich überwiegend so gestaltet sind, dass sich der deutsche und der polnische Leser bei der Lektüre der jeweils deutschen oder polnischen Version der Texte angesprochen fühlt. Dies betrifft sowohl die Deixis (vgl. (12), (13)) als auch andere Kategorien. Diese Strategie scheint in Texten neueren Datums (nach 2014) fortgesetzt zu werden. Als auffällig kann man bei der Untersuchung des Diskurses die Abmilderung der Expressivität der Aussage in einer der Sprachversionen der Texte feststellen. Diese an mehreren Techniken sichtbare Strategie ist mit dem Face-Paradigma von Goffman (1967) zu verbinden, dem zufolge im Paradigma Sprechende vs. Angesprochene das Gesicht des Gegenübers gewahrt wird. Dies drückt sich in einer inhaltlichen Profilierung aus, bei der die Radikalität der Darstellung mit Rücksicht auf den jeweiligen deutsch- oder polnischsprachigen Rezipienten abgeschwächt oder ganz neutralisiert wird.

(19) Niestety silne lobby niemieckich prywatnych klinik blokuje informowanie niemieckich pacjentów o takich możliwościach, a także prawach związanych z dyrektywą unijną. (G 1/2014 poln. online)

$\left(19^{`}\right)$ Leider verhindert eine überaus starke Krankenhauslobby in Deutschland, dass Patienten ausführlich über Behandlungsmöglichkeiten in Polen informiert werden. (G 1/2014 dt. online)

(20) Ostatnie zastrzelenie pacjenta w szpitalu na Śląsku było drastycznym sygnałem nieprawidłowego funkcjonowania tzw. sfery publicznej opieki zdrowotnej. (G 1/2014 poln. online)

$\left(20^{c}\right)$ Der jüngste Vorfall in Schlesien, wo Polizisten einen Krankenhauspatienten erschossen, zeigt drastisch, dass das öffentliche Gesundheitssystem aus den Fugen gerät. (G 1/2014 dt. online)

Die deutsche Fassung (19') mit Leider verbindert eine überaus starke Krankenhauslobby in Deutschland ist gegenüber dem rhetorisch aussagekräftigeren polnischen Text silne lobby niemieckich prywatnych klinik blokuje (19) etwas neutraler. Solche Eingriffe sind jedoch in den Textversionen und Passagen zu finden, in denen von Angesprochenen (hier von den Deutschen) die Rede ist.

In den untersuchten Texten auch älteren Datums, die hier aus Platzgründen nicht angeführt werden können, sind mehrere Beispiele für Translate von Passagen zu finden, in denen

20 Dies sind u. a. z. B. die Problematik der Vertriebenen, der Raketenschild, der Warschauer Aufstand, das Nord Stream-Projekt, die Kriminalität an der deutsch-polnischen Grenze etc. 
der Autor einer der Fassungen der Versuchung erliegt, die Ausdruckskraft der Aussage abzuschwächen (Bąk 2010a: 154).

(21) Niemieckie gazety alarmowały, że to Ukraina blokowała dostawy rosyjskiego gazu i stawiała rosyjskiemu dostawcy niewykonalne warunki. (I 1/2009: 26 poln./dt. Druck) ${ }^{21}$

(21') Deutsche Medien berichteten, dass die Ukraine russische Gaslieferungen blockierte und russischen Lieferanten unerfüllbare Bedingungen stellte. (I 1/2009: 27 poln./dt. Druck)

In dem Verständigungsdiskurs, der als Dialog zwischen den beiden Völkern aufgefasst werden kann, gelten die beiden Parteien als Sprechende sowie Angesprochene. Wie bereits in Bąk 2010a anhand älterer Belege vorgeschlagen und in 2016a und 2016b erörtert wurde, wird auch in der vorliegenden Arbeit dafür plädiert, die Hinwendungsstrategie mit dem Paradigma von Goffman in Verbindung zu setzen. ${ }^{22}$ Die Sprechenden und die Angesprochenen bilden eine Konstellation, in der das positive Gesicht des Gegenübers gewahrt wird.

Nicht selten grenzt - wie in $\left(13^{\circ}\right)$ - das Engagement der Diskursautoren (Autoren/Übersetzer) an Manipulation und verdient - was man beispielsweise in Bezug auf die Belege (22) und (23) sagen kann - einen translations- und diskurskritischen Blick (Bąk 2010a).

In der deutschen Fassung wird in (22') auf die Anführungszeichen verzichtet. Dieser Eingriff ist spürbar, denn dank dem orthographischen Mittel kann die polnische Phrase (,lieben') leichter als ironisch interpretiert werden. In $\left(23^{\circ}\right)$ ist wiederum eine andere fakultative Transposition zu verzeichnen. In der deutschen Fassung hat man überraschenderweise auf die rhetorische Frage verzichtet. Damit sowie mit weiteren Modifizierungen wird die kritische Aussage im polnischen Text in der deutschen Version neutralisiert, als ob man damit den deutschsprachigen Rezipienten hofieren möchte. ${ }^{23}$

(22) Być może dlatego teraz Putin tak „kocha” Niemcy, że chce o nich wiedzieć jak najwięcej. Przypomnijmy, Putin był szpiegiem KGB i rezydował w Dreźnie w latach 1985- 1990. (C 1/2014 poln. online)

(22') Vielleicht liebt Putin Deutschland deswegen so sehr, dass er alles über dieses Land erfahren möchte. Er war in den Jahren 1985-1990 als Spion für den KGB in Dresden stationiert. (C $1 / 2014 \mathrm{dt}$. online)

(23) Dzięki temu za kilka lat polskie firmy mogą być przejęte przez np. „niemiecką” spółkę, nie budząc sprzeciwu Polski. Pomysł dobry, godny biznesowego „Oskara” ale czy do zrealizowania przy „dystansie” jaki wobec Niemców i Unii Europejskiej ma część elity rządzącej Polską? (C 1/2014 poln. online)

(23') Dadurch könnten polnische Unternehmen in einigen Jahren zum Beispiel von einer „deutschen“ Firma übernommen werden, ohne dabei einen Einspruch Polens erwarten zu müssen. Dies ist keine schlechte Idee, die durchaus einen Wirtschafts-Oscar verdienen würde und die mit Blick auf die Distanz zwischen Deutschland (und der EU) und einem Teil der polnischen Regierungselite sogar Wirklichkeit werden könnte. (C 1/2014 dt. online)

21 Zu diesem sowie weiteren Belegen älteren Datums s. Bąk 2010a.

22 Zum Face-Paradigma von Goffman (1967) vgl. auch Mikołajczyk (2008), Bonacchi (2013), Bąk (2016b), Czachur $(2016,2018)$.

23 Aus Platzgründen können hier nicht die gesamten Passagen des Textes angeführt werden. Man muss hier allerdings hinzufügen, dass im Fall dieses Textabschnittes bereits im polnischen Text ein (unterstellter) deutscher Gedankengang bzw. eine diskursive Strategie aus deutscher Sicht realisiert wird. Dies führt bei der Übertragung ins Deutsche zu einer großen Komplexität. 
Die letztere Modifizierung $\left(23^{\circ}\right)$ verdient einen kritischen Blick, selbst wenn sie in guter Absicht der Diskursautoren und Übersetzer im Rahmen der (eigentlich positiv zu bewertenden) Hinwendungsstrategie vorgenommen worden sein sollte. Allerdings muss eingeräumt werden, dass in den untersuchten Korpustexten derartige Eingriffe nur selten anzutreffen sind.

Bei der Hinwendung werden die Sprachmittel gezielt eingesetzt, um den Blick aus einer bestimmten Perspektive zu vermitteln. Dies geschieht unter Einbeziehung der deutschen bzw. polnischen Grammatik, der Phraseologie, diverser pragmatischer Konventionen usw. und berücksichtigt wird zugleich die universelle Logik der Deixis einschließlich einiger weiterer Kategorien.

Im Zusammenhang mit der Schnittstelle zwischen der Übersetzungs- und Diskursanalyse, mit der man es im Falle der Analyse dieser Diskurse zu tun hat, sind zudem übersetzerische Adaptationsverfahren anzuführen, welche in der translationswissenschaftlichen Tradition oft in den Dichotomien ,Verfremden' vs. ,Verdeutschen' (Koller 2011: 297-303) und ,overt' vs. ,covert translation' (House 1997: 29) erörtert wird.

(24) Najdrożej w Europie płacimy za ropę i gaz, energię elektryczną. Mamy najwyższe w UE prowizje za płatność kartami. Droższe niż w bogatych Niemczech, i to nawet o kilkanaście procent, są u nas m.in.: woda mineralna, mąka pszenna, śmietana, mleko zagęszczone, wino. (F 1/2014 poln. online)

(24') In Polen zahlt man europaweit die höchsten Preise für Heizöl, Gas und Strom. Es gibt die europaweit höchsten Gebühren für Kartenzahlungen. Um viele Prozent teurer als im wohlhabenden Deutschland sind in Polen u.a. Mineralwasser, Weizenmehl, Sahne, Kondensmilch, Wein. (F 1/2014 dt. online)

(25) Zapowiedź katastrofy finansowej mieliśmy już w 2012 roku, gdy NFZ-etowi brakowało pieniędzy ze składek. (F 1/2014 poln. online)

(25) Der prophezeite finanzielle Zusammenbruch war 2012 fast da, als dem Nationalen Gesundheitsfonds das Geld ausging. (F 1/2014 dt. online)

(26) Liczenie przez prywatny sektor medyczny na przeciętnego Jana Kowalskiego, który z własnej kieszeni zapłaci za niewątpliwie bardzo dobrą opiekę medyczną, jest nieco iluzoryczne. (G 1/2014 poln. online)

(26) Die Hoffnungen auf den polnischen Durchschnittbürger, ${ }^{24}$ der aus eigener Tasche für die Behandlung bei privaten Ärzten zahlt, ist illusorisch. (G 1/2014 dt. online)

Solche Belege dokumentieren die „Logik“ bzw. Ordnung des bilingualen Verständigungsdiskurses, die durch die Rollen von Deutschen und Polen als Diskursakteure und Textrezipienten determiniert wird. Die beschriebenen inhaltlichen Adaptations- bzw. Anpassungsverfahren sind im Zusammenhang mit der diskursiven Hinwendungsstrategie zu betrachten.

Wie in bisherigen Arbeiten zu diesem Thema werden bei der Betrachtung des bilingualen Verständigungsdiskurses die Rollen der Diskursakteure mit Hilfe von grammatischen Kategorien zum einen der sprechenden, zum anderen der angesprochenen Person diskutiert, wodurch Aspekte der mündlichen Kommunikation (der Gesprächsforschung) mit einbezogen werden. Bei der Thematisierung von Problemen, die Deutsche und Polen in gegenseitiger

24 In anderen ähnlichen Kontexten kommt im Rahmen von Adaptationstechniken in der deutschen Fassung als Äquivalent für przeciętny Polak oder für Jan Kowalski der Ausdruck Otto-Normalverbraucher vor (Bąk 2020 im Druck). 
Berührung betreffen, tauschen die Deutschen und die Polen die Rollen der Sprechenden und Angesprochenen in den beiden Sprachversionen der Texte.

\subsection{Die Besprochenen im untersuchten Diskurs}

Im Gefüge der Sprechenden und Angesprochenen der Hinwendungsstrategie sind auch - was bereits der Beleg (16) suggeriert, Repräsentanten anderer Gesellschaften als Protagonisten der beschriebenen Geschehen präsent. Sie sind zumeist in der Rolle von Dritten, d.h. der Besprochenen zu verorten.

Modifizierungen der sprachlichen Gestalt, die in anderen angeführten Passagen mit Rücksicht auf den jeweiligen Rezipienten der Abmilderung oder Abschwächung der Expressivität dienen, sind (s. (22)) bei der Darstellung der Dritten viel seltener oder gar nicht anzutreffen ((16)-(18)), soweit sie nicht eine der unmittelbaren Parteien im Diskurs, d.h. die Sprechenden oder die Angesprochenen, betreffen. Die Repräsentanten anderer Gesellschaften werden im untersuchten Diskurs nicht als Partner oder Teilnehmer des dialogischen Diskurses profiliert. Wie bereits festgehalten (Bąk 2010a: 159), spielen sie hierbei vielmehr die Rolle der Besprochenen.

(27) Rosjanie „drezdeńczyka” Putina szpiegują i kupują co popadnie. Szczególnie chodzi im o energetykę, paliwa, telekomunikację i transport. Świetnie znający Niemców Putin, według doniesień prasowych, uważa Niemcy za swojego największego przyjaciela ale szpieguje go na potęgę. (C 1/2014 poln. online)

(27 ) Putin, der Deutschland hervorragend kennt, betrachtet das Land nach Presseberichten als seinen größten Freund. Dennoch lässt er dort in großem Umfang spionieren. Polen hingegen stellt für ihn eine Barriere zu einer besseren Welt, der EU, dar. (C 1/2014 dt. online)

Die Verbalisierung der Kritik zeichnet in solchen Fällen eine weitgehende Wörtlichkeit in den beiden Sprachfassungen aus. Aus Platzgründen können an dieser Stelle weitere Belege nicht angeführt werden (Bąk 2010a: 159-160, 2016a: 256-257). In anderen Analysen ist zu überprüfen, welche Aspekte und Themen im diskursiven Rahmen der Hinwendungsstrategie angesprochen und wie sie behandelt werden. Es sollte untersucht werden, ob andere Personen, Sachverhalte oder Kategorien, darunter historische und sozial-politische Entitäten, in dieser diskurs-paradigmatischen Rolle auch im Rahmen der Kategorie der/des Besprochenen profiliert werden.

\section{Schlussbemerkungen}

Die hier angeführten Belege bestätigen die im Rahmen des Projektes „Die Strategie der Hinwendung in bilingualen Verständigungsdiskursen" bisher gemachten Beobachtungen. In den untersuchten Texten erscheinen die Protagonisten des bilingualen Verständigungsdiskurses, Deutsche und Polen, die Adressaten bzw. Rezipienten der Texte sind, als Sprechende sowie Angesprochene des Diskurses. Es wird vorgeschlagen, in weiteren Untersuchungen 
die profilierte Konstellation der Hinwendung in Verbindung mit dem Face-Paradigma von Goffman (1967) an weiteren Belegen aus dem bilingualen Verständigungsdiskurses zu hinterfragen. Zu beleuchten sind besonders Aspekte des Framing im Zusammenhang mit der Problematik des durch den Diskurs aktivierten bzw. produzierten Wissens.

\section{Literatur}

\section{Quellen (mit Siglen)}

Region. Magazyn Polsko-Niemiecki. Deutsch-Polnisches Magazin. Kwartalnik/Quartalblatt, Nr. 1/2009 (= I 1/2009 poln./dt. Druck).

Region. Magazyn Polsko-Niemiecki. Deutsch-Polnisches Magazin. Kwartalnik/Quartalblatt, Nr. 2/2009. (= H 2/2009: poln./dt. Druck).

http://www.region.com.pl/pilkoszalony (= A 1/2016 poln. online) [30.10.2019].

http://www.region-europa.de/der-fubball-verruckte (= A 1/2016 dt. online) [30.10.2019].

http://www.region.com.pl/banksterzy (= B 1/2016 poln. online) [30.10.2019].

http://www.region-europa.de/bankster (= B 1/2016 dt. online) [30.10.2019].

http://www.region.com.pl/szpieguj-kupuj-szantazuj (= C 1/2014 poln. online) [30.10.2019].

http://www.region-europa.de/spionieren-kaufen-erpressen (= C 1/2014 dt. online) [30.10.2019].

http://www.region.com.pl/pamietniki-hitlera-falszerstwo-stulecia-1 (= D 1/2016 poln. online)

[30.10.2019].

http://www.region-europa.de/hitler-tagebucher-die-jahrhundert-falschung-1 (= D 1/2016 dt. online)

[30.10.2019].

http://www.region.com.pl/kto-tu-jest-niemcem (= E 1/2016 dt. online) [30.10.2019].

http://www.region-europa.de/wer-ist-hier-deutscher (= E 1/2016 dt. online) [30.10.2019].

http://www.region.com.pl/kraj-absurdow (=F 1/2014 poln. online) [30.10.2019].

http://www.region-europa.de/absurdes-land (= F 1/2014 dt. online) [30.10.2019].

http://www.region.com.pl/zdrowie-na-eksport (= G 1/2014 poln. online) [30.10.2019].

http://www.region-europa.de/gesundheit-fur-den-export (= G 1/2014 dt. online) [30.10.2019].

\section{Sekundärliteratur:}

Albrecht, Jörn (1973): Linguistik und Übersetzung. Tübingen: Niemeyer.

Bąk, Paweł (2010a): Deutsche und Polen in der zweisprachigen Presse am Beispiel der deutsch-polnischen Zeitung, Region' - ,Region' Gazeta polsko-niemiecka. Bemerkungen zur Strategie in Diskurs und Translation. In: Studia Germanica Resoviensia 8, 147-161.

Bąk, Paweł (2010b): ,Wir vergeben und bitten um Vergebung‘ po 44 latach. O dwujęzyczności debaty publicznej na przykładzie ,Oświadczenie Przewodniczącego Konferencji Episkopatu Polski i Przewodniczącego Konferencji Episkopatu Niemiec z okazji 70. rocznicy rozpoczęcia II wojny światowej 1 września 1939 roku'. [,Wir vergeben und bitten um Vergebung 44 Jahre später. Der bilinguale Charakter des öffentlichen Diskurses am Beispiel der ,Erklärung des Vorsitzenden der 
Deutschen Bischofskonferenz, Erzbischof Dr. Robert Zolltisch und des Vorsitzenden der Polnischen Bischofskonferenz, Erzbischof Dr. Józef Michalik, aus Anlass des 70. Jahrestages des Beginns des Zweiten Weltkriegs am 1. September 1939`]. In: Artur Czapiga, Zofia Czapiga (Hg.): Stowo i tekst w opisie porównawczym. [Wort und Text im Parallelvergleich]. Rzeszów: Wydawnictwo Uniwersytetu Rzeszowskiego, 11-24.

Bąk, Paweł (2016a): Die Sprechenden und Angesprochenen. Zur Strategie der Hinwendung in asymmetrischen Diskursen. In: Paweł Bąk, Bogusława Rolek (Hg.): Vom Wort zum Gebrauch. Wortbedeutung und ibre Eingebundenheit in Diskurse. Frankfurt/M.: Lang, 249-260.

Bąk, Paweł (2016b): Zur Strategie der Hinwendung in bilingualen deutsch-polnischen Diskursen. Vorüberlegungen zum integrativen Herangehen an die Schnittstelle von Translationswissenschaft und Diskursanalyse. In: Studia Translatorica 7, 11-23.

Bąk, Paweł (2018a): Gemeinsamkeiten von Translation und Diskursanalyse. Zur Erkenntnisperspektive in bilingualen Diskursen am Beispiel des Lehrwerks ,Europa. Nasza historia'/'Europa. Unsere Geschichte‘. In: Paweł Bąk und Bogusława Rolek (Hg.): Sprache und Translation. Rzeszów: Wydawnictwo Uniwersytetu Rzeszowskiego, 45-65.

Bąk, Pawel (2018b): Karl Dedecius: große Übersetzung der kleinen literarischen Form. In: Studia Translatorica 9. Die Botschaft der Bücher - Leben und Werk von Karl Dedecius, 123-137.

Bąk, Paweł (2019): Vom Fachlexem zum Unwort. Perspektive der Betrachtung. Perspektive der Erkenntnis. In: Linguistische Treffen in Wroctaw 15, 2019 (I), 29-40. DOI: 10.23817/lingtreff. 15-2.

Bąk, Paweł (2020 im Druck): Hinwendung zum Adressaten als diskursive Strategie am Beispiel der Zeitschrift, Region. In: Linguistische Treffen 18, 2020 (II).

Bonacchi, Silvia (2013): (Un)Höflichkeit. Eine kulturologische Analyse Deutsch-Italienisch-Polnisch. Frankfurt/M.: Lang.

Brown, Penelope / Levinson, Stephen C. (1987): Politeness: some universals in language usage. Cambridge: Cambridge University Press.

Busse, Dietrich (2012): Frame-Semantik. Ein Kompendium. Berlin, Boston: de Gruyter.

Busse, Dietrich (2015): Sprachverstehen und Textinterpretation Grundzüge einer verstehenstheoretisch reflektierten interpretativen Semantik. Wiesbaden: Springer VS.

Czachur, Waldemar (2016): Sprachliche Beziehungsgestaltung in den deutsch-polnischen Relationen. Eine linguistische Analyse des Briefes der polnischen Bischöfe an die deutschen Bischöfe aus dem Jahre 1965. In: Jianhua Zhu, Jin Zhao, Michael Szurawitzki (Hg.): Akten des XIII. Internationalen Germanistenkongresses Shanghai 2015. Bd. 3: Germanistik zwischen Tradition und Innovation. Frankfurt/M.: Lang, 33-37.

Czachur, Waldemar (2018): Symbolisches Gleichgewicht und internationales Beziehungsmanagement. Eine linguistische Analyse des Briefes der polnischen Bischöfe an die deutschen Bischöfe aus dem Jahre 1965. In: Paweł Bąk, Bogusława Rolek (Hg.): Vom Wort zum Gebrauch. Wortbedeutung und ihre Eingebundenheit in Diskurse. Frankfurt/M.: Lang, 261-277.

Fillmore, Charles J. (1977): Scenes-and-Frames Semantics. In: Antonio Zampolli (Hg.): Linguistic Structures Processing Vol. 5. Amsterdam u. a.: North Holland, 55-81.

Fleischer, Wolfgang (1997): Phraseologie der deutschen Gegenwartssprache. Tübingen: Niemeyer. Foucault, Michel (1981): Archäologie des Wissens. (Dt.: Ulrich Köppen). Frankfurt/M.: Suhrkamp. Fraas, Claudia (2013): Frames - ein qualitativer Zugang zur Analyse von Sinnstrukturen in der OnlineKommunikation. In: Barbara Frank-Job, Alexander Mehler, Tilmann Sutter (Hg.): Die Dynamik 
sozialer und sprachlicher Netzwerke: Konzepte, Methoden und empirische Untersuchungen an Beispielen des $W W W$. Wiesbaden: Springer VS, 259-283.

Fraas, Claudia / Meier, Stefan (2013): Multimodale Stil- und Frameanalyse - Methodentriangulation zur medienadäquaten Untersuchung von Online-Diskursen. In: Kersten Sven Roth, Carmen Spiegel (Hg.): Angewandte Diskurslinguistik. Felder, Probleme, Perspektiven. Berlin: Akademie Verlag, $135-162$.

Goffman, Erving (1967): Interaction Rituals. New York: Pantheon Books.

Grice, Herbert Paul (1975): Logic and Conversation. In: Peter Cole, Jerry Morgan (Hg.): Syntax and Semantics. 3: Speech Acts. New York: Academic Press, 41-58.

House, Juliane (1997): Translation Quality assessment: A Model Revisited. Tübingen: Narr.

Koller, Werner (2011): Einführung in die Übersetzungswissenschaft. Tübingen: Francke.

Mikołajczyk, Beata (2008): Wyrażenia znieważające jako leksykalne środki realizacji aktów zagrażających twarzy na przykładzie języka niemieckiego i polskiego [Schimpfwörter als lexikalische Ausführungsmittel gesichtsbedrohender Akte am Beispiel des Deutschen und des Polnischen]. In: Andrzej Kątny (Hg.): Kontakty językowe i kulturowe w Europie. [Sprach- und Kulturkontakte in Europa]. Gdańsk: Wydawnictwo Uniwersytetu Gdańskiego, 186-197.

Pawłowski, Grzegorz (2017): Fachlexeme in Konstruktion. Linguistischer Beitrag zur Erkenntnisarbeit. Frankfurt/M.: Lang.

Pędzisz, Joanna (2012): Diskursthema und Themen im Diskurs. Zur thematischen Profilierung der diskursiven Wirklichkeit. In: tekst i dyskurs - text und diskurs 5, 231-247.

Schreiber, Michael (1993): Übersetzung und Bearbeitung. Zur Differenzierung und Abgrenzung des Übersetzungsbegriffs. Tübingen: Narr.

Spitzmüller, Jürgen / Warnke, Ingo H. (2011): Diskurslinguistik. Eine Einführung in Theorien und Methoden der transtextuellen Sprachanalyse. Berlin, Boston: de Gruyter.

Vinay, Jean-P. / Darbelnet, Jean (1958): Stylistique comparée du français et de l'anglais. Méthode de traduction. Paris: Didier.

Völker, Daniel (2017): Kommunikation im Krisenmodus: Konzeption des Strategischen Framing am Beispiel der Finanzkrise 2008/09. Wiesbaden: Springer.

Ziem, Alexander (2013): Syntaktische Konstruktionen als diskursive Muster Krisen in der medienvermittelten Öffentlichkeit. In: Jens Maeße (Hg.): Ökonomie, Diskurs, Regierung. Interdisziplinäre Perspektiven. Wiesbaden: Springer, 148-166. 